\title{
Theory of nucleon transfer in the dynamical two-center shell model
}

\author{
Jae Y. Park \\ Department of Physics, North Carolina State University, Raleigh, North Carolina 27607 \\ Werner Scheid \\ Institut für Theoretische Physik der Justus-Liebig-Universität Giessen, West-Germany \\ Walter Greiner \\ Institut für Theoretische Physik der Johann-Wolfgang-Goethe-Universität Frankfurt/Main, West-Germany \\ (Received 1 November 1978)
}

\begin{abstract}
The theory of nucleon transfer in heavy ion reactions is formulated on the basis of the molecular particlecore model for a system consisting of two cores and one extracore nucleon. The extracore nucleon is described by the molecular wave functions of the asymmetric two-center shell model. The cores, which are assumed to be collectively excitable, are treated with vibrator-rotator models. Potentials for shape polarization are contained in the asymmetric two-center shell model and the interaction between the cores. The excitation and transfer of the extracore nucleon is induced by the radial and rotational couplings. The coupled channel equations, which include the recoil effects in first approximation, are derived in a form suitable for numerical calculations of cross sections.
\end{abstract}

NNUCLEAR REACTIONS Heavy ion scattering, theory of nucleon transfer, molecular wave functions, two-center shell model, collective and singleparticle excitation.

\section{INTRODUCTION}

Although collective molecular resonances have been observed in various heavy ion systems, such as ${ }^{12} \mathrm{C}+{ }^{12} \mathrm{C}$, molecular single-particle effects are not well 'established (for a review see Ref. 1). In this paper we study molecular single-particle ef fects in the scattering of nuclei which can be decomposed into a core and a loosely bound nucleon. For simplicity, we restrict our investigations to systems with one extracore nucleon. Examples of such systems are ${ }^{12} \mathrm{C}+{ }^{13} \mathrm{C}$ for identical cores and ${ }^{13} \mathrm{C}+{ }^{16} \mathrm{O}$ for different cores. The main purpose of our paper is to derive the elements for the molecular description of the excitation and transfer of the extracore nucleon.

The theory of nuclear molecular single-particle effects was first initiated with the symmetric and asymmetric two-center shell models ${ }^{2}$ (STCSM, ATCSM) which are mostly applied to calculate real nucleus-nucleus potentials. ${ }^{3}$ Dynamic treatments of molecular single-particle motion in nucleus-nucleus collisions were studied by Park et al., ${ }^{4}$ von Oertzen and Nörenberg, ${ }^{5}$ Becker et al., ${ }^{6}$ Matveenko and Lovas, ${ }^{7}$ and Terlecki et al. ${ }^{8}$ Also, molecular wave functions were used to describe polarization effects in proton transfer reactions. ${ }^{9}$

One major difference between our theory and the work in Refs. 6 and 7 lies in the different definition of the rotating coordinate systems. In Refs. 6 and 7 , one defines the $z^{\prime}$ axis of the rotating system by the direction of the core-core distance. This definition is commonly used in atomic physics where the rotating system is fixed by the position of the nuclear centers. However, in nuclear physics the mass ratio between the extracore nucleon and the cores does not allow us to equate the core-core distance with the relative coordinate. Therefore, in Refs. 4 and 8 we have defined the position of the intrinsic coordinate system with the true relative coordinate, and consequently avoided all the complications arising in the asymptotic behavior of the wave functions as discussed in Refs. 6 and 7 where the core-core distance has been used.

Further, we assume that the center distance in the TCSM should be taken as the relative coordinate instead of the core-core distance. This assumption becomes most obvious for an increasing number of extracore nucleons. The TCSM potential is generated as the mean field of all the nucleons and not just the core nucleons only. Therefore, the center distance has to be related to the relative coordinate of all the nucleons.

This work is a generalization of the paper by Terlecki et al. ${ }^{8}$ on the ${ }^{13} \mathrm{C}-{ }^{13} \mathrm{C}$ reaction in that the extracore nucleons can be transferred and that the cores can be collectively excited. Both effects, which we have not yet studied, play a role in the ${ }^{13} \mathrm{C}-{ }^{13} \mathrm{C}$ reaction. In order to avoid the 
complexity in the theory due to the antisymmetrization of the extracore nucleons, we have restricted our formulation to the case of one extracore nucleon only. However, this assumption is not very restrictive and can be removed straightforwardly.

In Sec. II we formulate the coordinates and the Hamiltonian describing the two cores and the loosely bound nucleon. The dynamics of the cores is treated by collective surface coordinates which have to be properly defined as the nuclei overlap. Hence, the low-energy spectra of the cores are explained in the framework of vibrator or rotator-vibrator models. Analogously to the strong coupling model (Nilsson model) we assume in addition that the ATCSM potential depends on the collective surface coordinates. Therefore, the model contains two types of polarization of the nuclear shapes. The first type arises due to the overlap of the nuclei and depends on the relative distance of the nuclei, whereas the second type is caused by collective transitions in the cores via the dynamics of the scattering process. Since the relative coordinate between the nuclei changes after the transfer of the extracore nucleon, the Hamiltonian has different forms for the direct and transfer channels.

Section III presents the wave functions, their asymptotic behavior and symmetries for identical cores. In Sec. IV we construct the coupled channel equations for the relative wave functions. The coupled equations are written very explicitly so that practical numerical calculations can be started. For this purpose we expand the recoil terms up to the first order in the difference between the relative coordinates of the two fragmentations. It is shown that the radial and rotational couplings are important mechanisms for the excitation and transfer of the extracore nucleon.

\section{THE MODEL}

We consider the scattering of a system of two nuclei consisting of two cores with $C_{1}$ and $C_{2}$

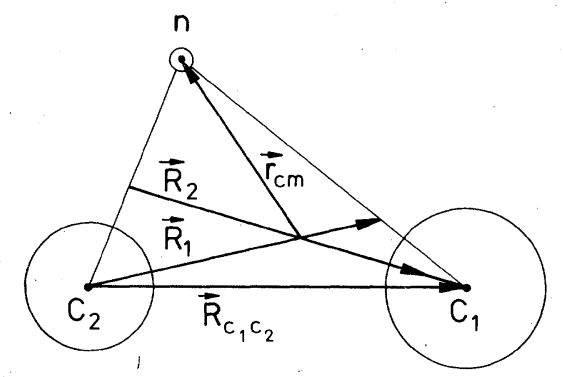

FIG. 1. Definition of the various coordinates of the particle-core model. nucleons and one extracore nucleon. The extracore nucleon can be transferred between the cores. Such systems are, for example, ${ }^{13} \mathrm{C}+{ }^{12} \mathrm{C}$, ${ }^{13} \mathrm{C}+{ }^{16} \mathrm{O}$, and ${ }^{12} \mathrm{C}+{ }^{17} \mathrm{O}$. For simplicity we restrict the theory to systems with one extracore nucleon. The extension to systems with more extracore nucleons is straightforward, though one needs to antisymmetrize the wave functions of the extracore nucleons. The following reaction channels are possible:

$$
\begin{array}{ll}
s=1 \quad & \left(C_{1}+1\right)+C_{2}, \\
& \left(C_{1}+1\right) *+C_{2}, \\
& \left(C_{1}+1\right)+C_{2}^{*}, \\
& \left(C_{1}+1\right)^{*}+C_{2}^{*}, \\
s=2 \quad & C_{1}+\left(C_{2}+1\right), \\
& C_{1}^{*}+\left(C_{2}+1\right), \\
& C_{1}+\left(C_{2}+1\right) *, \\
& C_{1}^{*}+\left(C_{2}+1\right)^{*},
\end{array}
$$

The channels with $s=1$ and $s=2$ consist of nuclei in the fragmentations $A_{1}=C_{1}+1, A_{2}=C_{2}$ and $A_{1}=C_{1}, A_{2}=C_{2}+1$, respectively. In the excited states of the nuclei both the cores and the extracore nucleon may be excited. The excited states of the cores are assumed to be describable by collective models such as rotation or vibration models. ${ }^{10}$ The states of the extracore nucleon are obtained from the asymmetric two-center shell model suitable for the fragmentations with $A_{1}$ and $A_{2}$ nucleons. ${ }^{11}$

\section{A. Coordinates}

We denote the laboratory coordinates of the core centers with $\vec{R}_{C_{1}}$ and $\vec{R}_{C_{2}}$ and the laboratory coordinate of the extracore nucleon by $\vec{r}_{1}$. As shown in Fig. 1, we introduce the center-of-mass coordinate of the system with $A\left(=C_{1}+C_{2}+1\right)$ nucleons,

$$
\overrightarrow{\mathrm{R}}_{\mathrm{c} . \mathrm{m} .}=\frac{1}{A}\left(C_{1} \overrightarrow{\mathrm{R}}_{C_{1}}+C_{2} \overrightarrow{\mathrm{R}}_{C_{2}}+\overrightarrow{\mathrm{r}}_{1}\right)
$$

the coordinate of the extracore nucleon measured with respect to the center of mass

$$
\overrightarrow{\mathbf{r}}_{\mathrm{c} . \mathrm{m} .}=\overrightarrow{\mathrm{r}}_{1}-\overrightarrow{\mathrm{R}}_{\mathrm{c} . \mathrm{m} .},
$$

and the relative coordinates between the nuclear centers in the channels with $s=1$ and $s=2$,

$$
\begin{aligned}
& \overrightarrow{\mathrm{R}}_{s=1}=\frac{1}{C_{1}+1}\left(C_{1} \overrightarrow{\mathrm{R}}_{C_{1}}+\overrightarrow{\mathrm{r}}_{1}\right)-\overrightarrow{\mathrm{R}}_{C_{2}}, \\
& \overrightarrow{\mathrm{R}}_{s=2}=\overrightarrow{\mathrm{R}}_{C_{1}}-\frac{1}{C_{2}+1}\left(C_{2} \overrightarrow{\mathrm{R}}_{C_{2}}+\overrightarrow{\mathrm{r}}_{1}\right) .
\end{aligned}
$$

Between the coordinates defined in Eqs. (4) and 
(5), the following relations hold:

$$
\begin{aligned}
& \overrightarrow{\mathrm{R}}_{1}=\overrightarrow{\mathrm{R}}_{2}+\frac{1}{\left(C_{1}+1\right) C_{2}}\left[\left(C_{1}-C_{2}\right) \overrightarrow{\mathrm{R}}_{2}+A \overrightarrow{\mathrm{r}}_{\mathrm{c} . \mathrm{m} .}\right], \\
& \overrightarrow{\mathrm{R}}_{2}=\overrightarrow{\mathrm{R}}_{1}-\frac{1}{\left(C_{2}+1\right) C_{1}}\left[\left(C_{1}-C_{2}\right) \overrightarrow{\mathrm{R}}_{1}+A \overrightarrow{\mathrm{r}}_{\mathrm{c} . \mathrm{m} .}\right] .
\end{aligned}
$$

For equal cores $C_{1}=C_{2}=C$ these formulas reduce to a single expression:

$$
\overrightarrow{\mathrm{R}}_{1}=\overrightarrow{\mathrm{R}}_{2}+\frac{2 C+1}{(C+1) C} \overrightarrow{\mathrm{r}}_{\text {c.m. }} .
$$

In the calculation of the matrix elements the volume element has to be inserted as

$$
d \tau_{s}=\left(\frac{C_{s}+1}{C_{s}}\right)^{3} d^{3} \overrightarrow{\mathrm{R}}_{\mathrm{c} . \mathrm{m} .} d^{3} \overrightarrow{\mathrm{R}}_{s} d^{3} \overrightarrow{\mathrm{r}}_{\mathrm{c} . \mathrm{m} .} \text {. }
$$

The dynamics of the cores is described by multipole coordinates $\alpha_{\lambda \mu}^{(1)}$ and $\alpha_{\lambda \mu}^{(2)}$ which define the shape of the cores 1 and 2 with respect to the laboratory axes. ${ }^{10}$ In most applications the quadrupole deformation of the nuclear shapes is the important one which can be treated in the framework of the vibrator or rotator models. Restricting the further considerations to quadrupole shapes only, we define the nuclear surfaces asymptotically by

$$
\mathbb{R}_{i}=R_{0 i}\left(1+\sum_{\mu} \alpha_{2 \mu}^{(i)} Y_{2 \mu}^{*}\left(\theta_{i}, \varphi_{i}\right)\right), i=1,2 .
$$

Here, $R_{0 i}$ denotes the nuclear radii. In the overlap region the nuclear surface has to be defined in accordance with the corresponding equipotential surface of the asymmetric two-center shell model. The deformation of this equipotential surface can be related to the coordinates $\alpha_{2 \mu}^{(i)}$ as shown for the symmetric two-center shell model by Fink et $a l .{ }^{12}$

\section{B. Kinetic energy}

The kinetic energy is given in the center-ofmass system by

$$
T=T_{C 1}+T_{C_{2}}+\frac{p_{1}^{2}}{2 M}+T_{\text {coll }}\left(\pi_{2 \mu}^{(i)}\right)-T_{\text {c.m. }} \cdot
$$

The kinetic energies are those of the centers of the cores, of the extracore nucleon, and of the collective degrees of freedom of the cores. We transform the Hamiltonian for each fragmentation $s$ to a different rotating coordinate system with the $z^{\prime}$ axis in the direction of $\overrightarrow{\mathrm{R}}_{s}$. The rotating coordinate systems are fixed with respect to the laboratory system by the Euler angles $\varphi_{s}$, $\theta_{s}$ (spherical polar angles of $\overrightarrow{\mathrm{R}}_{s}$ ), and $\psi_{s}$. The angle $\psi_{s}$ around the $z^{\prime}$ axis has no physical sig nificance and does not affect the results. After the transformation to the rotating coordinate systems the kinetic energy, given in (10), may be written for the fragmentation $s$ as

$$
\begin{aligned}
T=T_{s}= & -\frac{\hbar^{2}}{2 \mu_{s} R_{s}}\left(\frac{\partial}{\partial R_{s}}+D_{s}\right)^{2} R_{s}+\frac{1}{2 M}\left(1-\frac{1}{C_{s}+1}\right) \overrightarrow{\mathrm{p}}_{\mathrm{c} . \mathrm{m} .}^{\prime 2} \\
& +\frac{1}{2 \mu_{s} R_{s}{ }^{2}}\left(\overrightarrow{\mathrm{I}}\left(\varphi_{s}, \vartheta_{s}, \psi_{s}\right)-\overrightarrow{\mathrm{J}}_{s}-\overrightarrow{\mathrm{J}}_{\mathrm{coll}}\left(\pi_{2 \mu}^{\prime(i)}, \alpha_{2 \mu}^{\prime(i)}\right)\right)^{2} \\
& +T_{\text {coll }}^{\prime}\left(\pi_{2 \mu}^{\prime(i)}\right),
\end{aligned}
$$

where

$$
\begin{aligned}
& D_{1}=\frac{C_{2}}{A} \frac{\partial}{\partial z_{\mathrm{c} . \mathrm{m} .}^{\prime}}, \quad D_{2}=-\frac{C_{1}}{A} \frac{\partial}{\partial z_{\mathrm{c} . \mathrm{m} .}^{\prime}}, \\
& \overrightarrow{\mathrm{J}}_{1}=\left(\overrightarrow{\mathrm{r}}_{\mathrm{c} . \mathrm{m} \cdot}^{\prime}-\frac{C_{2}}{A} R_{1} \overrightarrow{\mathrm{e}}_{\boldsymbol{z}^{\prime}}\right) \times \overrightarrow{\mathrm{p}}_{\mathrm{c} . \mathrm{m} .}^{\prime}+\overrightarrow{\mathrm{s}}^{\prime}, \\
& \overrightarrow{\mathrm{J}}_{2}=\left(\overrightarrow{\mathrm{r}}_{\mathrm{c} . \mathrm{m} .}^{\prime}+\frac{C_{1}}{A} R_{2} \overrightarrow{\mathrm{e}}_{z^{\prime}}\right) \times \overrightarrow{\mathrm{p}}_{\mathrm{c} . \mathrm{m} .}^{\prime}+\overrightarrow{\mathrm{s}}^{\prime}, \\
& \mu_{1}=\left(C_{1}+1\right) C_{2} M / A, \quad \mu_{2}=C_{1}\left(C_{2}+1\right) M / A .
\end{aligned}
$$

Here, $\vec{I}, \vec{J}_{s}$, and $\vec{J}_{\text {coll }}$ are, respectively, the operators of the total angular momentum including the nucleon spins, the angular momentum of the extracore nucleon measured with respect to the nuclear centers, and the total angular momentum of the quadrupole degrees of freedom. The angular momenta and the linear momenta $\overrightarrow{\mathrm{p}}_{\mathrm{c} . \mathrm{m}}^{\prime}$ and $\pi_{2 \mu}^{\prime(i)}$ are written with respect to the rotating coordinate system. The reduced masses for the fragmentation $s$ are abbreviated by $\mu_{s}$ with $M$ as the nucleon mass. The details of the transformation to the rotating coordinate system are given in Refs. 8 and 10.

\section{Hamiltonian}

The Hamiltonian for the fragmentation $s$ is assumed to be as follows:

$$
\begin{aligned}
H= & T_{s}+V_{\text {coll }}\left(\alpha_{2 \mu}^{(i)}\right)+U\left(\overrightarrow{\mathrm{R}}_{C_{1}}-\overrightarrow{\mathrm{R}}_{C 2}, \alpha_{2 \mu}^{(i)}\right) \\
& +i W\left(R_{s}, \overrightarrow{\mathrm{I}}^{2}, E\right)+V_{s}\left(\overrightarrow{\mathrm{r}}_{\mathrm{c} . \mathrm{m} .}^{\prime}, \overrightarrow{\mathrm{p}}_{\mathrm{c} . \mathrm{m} .}^{\prime}, \overrightarrow{\mathrm{s}}^{\prime}, \alpha_{2 \mu}^{\prime(i)}, R_{s}\right) .
\end{aligned}
$$

The potential $V_{\text {coll }}$ is the asymptotic potential energy of the quadrupole degrees of freedom $\left(R_{s} \rightarrow \infty\right)$. The potential $U$ represents the interaction between the cores and depends only on the core-core separation distance and the deformation parameters of the nuclear surface. The absorptive potential $W$ is due to all the channels which are not explicitly treated and is assumed to be a function of the relative coordinate, the total angular momentum and energy. The fifth term $V_{s}$ is the potential of the asymmetric twocenter shell model (ATCSM) in the fragmentation $s$.

For the core-core potential we make a multipole expansion up to first order of the deforma- 
tion parameters $\alpha_{2 \mu}^{(i)}$ :

$$
\begin{aligned}
U=U_{0}\left(R_{C_{1} C_{2}}\right)+\sum_{\mu} & {\left[I_{2}^{(1)}\left(R_{C_{1} C_{2}}\right) \alpha_{2 \mu}^{(1)}\right.} \\
+ & \left.I_{2}^{(2)}\left(R_{C_{1} C_{2}}\right) \alpha_{2 \mu}^{(2)}\right] Y_{2 \mu}^{*}\left(\Omega_{C_{1} C_{2}}\right) .
\end{aligned}
$$

In prinicple, it is possible to describe the nucleus-nucleus motion by the core-core distance and single-particle coordinates as shown by Becker et al. ${ }^{6}$ But such a procedure leads to problems in the asymptotic behavior of the wave functions for large internuclear distances. Only with the true relative coordinates $\vec{R}_{s}$ does the translational motion decouple asymptotically from the intrinsic nuclear degrees of freedom. There- fore, we describe the nucleus-nucleus motion with the relative coordinates $\vec{R}_{s}$, defined in Eqs. (5). But then we have to express the core-core potential as a function of the relative coordinates. The core-core distance can be written as

$$
\begin{aligned}
\overrightarrow{\mathrm{R}}_{C_{1} C_{2}}=\overrightarrow{\mathrm{R}}_{C_{1}}-\overrightarrow{\mathrm{R}}_{C_{2}} & =\left(1+C_{2} / C_{1} A\right) \overrightarrow{\mathrm{R}}_{1}-\overrightarrow{\mathrm{r}}_{\mathrm{c} \cdot \mathrm{m} .} / C_{1} \\
& =\left(1+C_{1} / C_{2} A\right) \overrightarrow{\mathrm{R}}_{2}+\overrightarrow{\mathrm{r}}_{\mathrm{c} \cdot \mathrm{m} .} / C_{2} .
\end{aligned}
$$

Since $\overrightarrow{\mathrm{R}}_{C_{1}}-\overrightarrow{\mathrm{R}}_{C_{2}} \simeq \overrightarrow{\mathrm{R}}_{1} \simeq \overrightarrow{\mathrm{R}}_{2}$ for $C_{1}, C_{2}>1$, we may expand the potential in the difference between the relative and core-core distance. For the fragmentation $s=1$ we obtain in first order of the difference

$$
\begin{aligned}
U_{s=1}= & {\left[1+\frac{1}{C_{1}}\left(\frac{C_{2}}{A} R_{1}-z_{\text {c.m. }}^{\prime}\right) \frac{\partial}{\partial R_{1}}\right]\left\{U_{0}\left(R_{1}\right)+\left(\frac{5}{4 \pi}\right)^{1 / 2}\left[I_{2}^{(1)}\left(R_{1}\right) \alpha_{20}^{(1)}+I_{2}^{(2)}\left(R_{1}\right) \alpha_{20}^{\prime(2)}\right]\right\} } \\
& +\frac{1}{C_{1} R_{1}} \sqrt{5} r_{\text {c.m. }}\left[\left(I_{2}^{(1)}\left(R_{1}\right) \alpha_{21}^{\prime(1)}+I_{2}^{(2)}\left(R_{1}\right) \alpha_{21}^{\prime(2)}\right) Y_{1-1}\left(\vartheta_{\mathrm{c} . \mathrm{m} .,}^{\prime} \varphi_{\mathrm{c} . \mathrm{m} .}^{\prime}\right)\right. \\
& \left.+\left(I_{2}^{(1)}\left(R_{1}\right) \alpha_{2-1}^{\prime(1)}+I_{2}^{(2)}\left(R_{1}\right) \alpha_{2-1}^{(2)}\right) Y_{11}\left(\vartheta_{\mathrm{c} . \mathrm{m} .,}^{\prime} \varphi_{\mathrm{c} . \mathrm{m} .}^{\prime}\right)\right]
\end{aligned}
$$

A similar expression results for $U_{s=2}$. In most applications it is sufficient to replace the core-core distance in Eq. (13) by the relative coordinates $\vec{R}_{1}$ and $\vec{R}_{2}$. The additional terms in Eq. (15) are due to the difference between the center-of-mass coordinates of the nuclei and those of the cores and are small because of the common factor $1 / C_{1}$.

It is presumed that the potential $V_{s}$ of the ATCSM in Eq. (12) represents the mean field of all nucleons and, therefore, is generated by the core nucleons and the extracore nucleons. Hence, it is realistic to assume that the centers of the ATCSM coincide with the centers of mass of the nuclei in the fragmentations $s=1,2$. In the rotating coordinate systems the centers lie at

$$
\begin{aligned}
& s=1: \quad z^{\prime}=\frac{C_{2}}{A} R_{1} ;-\frac{C_{1}+1}{A} R_{1}, \\
& s=2: \quad z^{\prime}=\frac{C_{2}+1}{A} R_{2} ;-\frac{C_{1}}{A} R_{2} .
\end{aligned}
$$

The potential of the ATCSM can be written, up to terms linear in the deformation coordinates $\alpha_{2 \mu}^{\prime(i)}$, as

$$
V_{s}=V_{0 s}\left(\overrightarrow{\mathrm{r}}_{\mathrm{c} . \mathrm{m} .}^{\prime}, R_{s}\right)+V_{l^{2}, s}+V_{l s, s}-k_{s} \sum_{\mu}\left(g_{s}\left(z_{\mathrm{c}, \mathrm{m} .}^{\prime}\right) \alpha_{2 \mu}^{\prime(1)}+g_{s}\left(-z_{\mathrm{c} . \mathrm{m} .}^{\prime}\right) \alpha_{2 \mu}^{\prime(2)}\right) Y_{2 \mu}^{*}\left(\vartheta_{\mathrm{c} . \mathrm{m} .,}^{\prime} \varphi_{\mathrm{c} . \mathrm{m} .}^{\prime}\right)\left(\overrightarrow{\mathrm{r}}_{\mathrm{c} . \mathrm{m}}^{\prime} \operatorname{grad}^{\prime}\right) V_{o s}\left(\overrightarrow{\mathrm{r}}_{\mathrm{c}, \mathrm{m} ., R_{s}}^{\prime} R_{s}\right),
$$

with

$$
g_{s}(z)=\left\{\begin{array}{l}
1 \text { for } z \gg 0 \\
0 \text { for } z \ll 0
\end{array}\right.
$$

Here $V_{l^{2}, s}$ is an $l^{2}$-type potential and $V_{l s, s}$ the spin-orbit potential. Their precise definitions are given in Ref. 11, where the details about the potential $V_{0 s}$ are also discussed. The last term couples the singleparticle motion with the quadrupole degrees of freedom. The coupling constants $k_{s}$ may be obtained by fitting the asymptotic energy spectra of the nuclei. The function $g_{s}(z)$ is defined as a smeared-out step function in order to have a smooth transition of the nuclear surface from the coordinates $\alpha_{2 \mu}^{\prime(1)}$ to $\alpha_{2 \mu}^{\prime(2)}$ at the plane $z^{\prime}=0$. The Hamiltonian (12) can be rewritten as follows:

$$
H(s)=H_{\text {coll }}\left(\pi_{2 \mu}^{\prime(i)}, \alpha_{2 \mu}^{\prime(i)}\right)+h_{\mathrm{ATCSM}}\left(\overrightarrow{\mathrm{r}}_{\mathrm{c} . \mathrm{m} .}^{\prime}, s, R_{s}\right)+\widetilde{T}_{s}+U_{0}\left(R_{s}\right)+i W\left(R_{s}, \overrightarrow{\mathrm{I}}^{2}, E\right)+W_{\text {coup }}\left(R_{s}, \alpha_{2 \mu}^{\prime(i)}, \overrightarrow{\mathrm{r}}_{\mathrm{c} . \mathrm{m} .}^{\prime}\right) .
$$

Here, we have used the abbreviations:

$$
H_{\text {coll }}=H_{\text {coll , } 1}\left(\pi_{2 \mu}^{\prime(1)}, \alpha_{2 \mu}^{\prime(1)}\right)+H_{\text {coll , } 2}\left(\pi_{2 \mu}^{\prime(2)}, \alpha_{2 \mu}^{\prime(2)}\right),
$$




$$
\begin{aligned}
& h_{\mathrm{ATCSM}}=\frac{1}{2 M}\left(1-\frac{1}{C_{s}+1}\right) \overrightarrow{\mathrm{p}}_{\mathrm{c} . \mathrm{m} .}^{\prime 2}+V_{0 s}\left(\overrightarrow{\mathrm{r}}_{\mathrm{c} . \mathrm{m} .}^{\prime}, R_{s}\right)+V_{l 2, s}+V_{l s, s} \\
& \dot{T}_{s}=-\frac{\hbar^{2}}{2 \mu_{s} R_{s}}\left(\frac{\partial}{\partial R_{s}}+D_{s}\right)^{2} R_{s}+\frac{1}{2 \mu_{s} R_{s}^{2}}\left(\overrightarrow{\mathrm{I}}_{s}-\overrightarrow{\mathrm{J}}_{s}-\overrightarrow{\mathrm{J}}_{\mathrm{col1}}\right)^{2} \text {, } \\
& W_{\text {coup }}(s=1)=\left[1+\frac{1}{C_{1}}\left(\frac{C_{2}}{A} R_{1}-z_{\text {c.m. }}^{\prime}\right) \frac{\partial}{\partial R_{1}}\right]\left(\frac{5}{4 \pi}\right)^{1 / 2}\left[I_{2}^{(1)}\left(R_{1}\right) \alpha_{20}^{(1)}+I_{2}^{(2)}\left(R_{1}\right) \alpha_{20}^{\prime(2)}\right]+\frac{1}{C_{1}}\left(\frac{C_{2}}{A} R_{1}-z_{\mathrm{c} . \mathrm{m} .}^{\prime}\right) d U_{0}\left(R_{1}\right) / d R_{1} \\
& +\frac{\sqrt{5} r_{c . m .}}{C_{1} R_{1}}\left[\left(I_{2}^{(1)}\left(R_{1}\right) \alpha_{21}^{(1)}+I_{2}^{(2)}\left(R_{1}\right) \alpha_{21}^{\prime(2)}\right) Y_{1-1}\left(\vartheta_{\mathrm{c} . \mathrm{m},}^{\prime} \varphi_{\mathrm{c} . \mathrm{m}}^{\prime}\right)\right. \\
& \left.+\left(I_{2}^{(1)}\left(R_{1}\right) \alpha_{2 \infty 1}^{(1)}+I_{2}^{(2)}\left(R_{1}\right) \alpha_{2-1}^{\prime(2)}\right) Y_{11}\left(\vartheta_{\mathrm{c} . \mathrm{m},}^{\prime}, \varphi_{\mathrm{c} . \mathrm{m} .}^{\prime}\right)\right]
\end{aligned}
$$

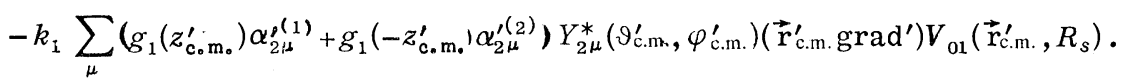

Analogously, we have $W_{\text {coup }}(s=2)$. Mainly the operators $T_{s}$ and $W_{\text {coup }}$ couple the various degrees of freedom. We note that the Hamiltonian (18) depends on the special fragmentation $s$.

\section{WAVE FUNCTIONS}

The wave functions for solving the scattering problem $H \psi=E \psi$ are given in the laboratory system by the ansatz (Park et $a l_{.}^{4}$ )

$\psi_{I M}=\sum_{s \alpha l J} R_{s \alpha l J I}\left(R_{s}\right)\left[i^{l} Y_{l}\left(\vartheta_{s}, \varphi_{s}\right) \otimes \Phi_{s \alpha J}\right]_{M}^{[I]}$.

Here $R_{\text {s } l_{l J I}}\left(R_{s}\right)$ represents the wave function describing the relative motion of the colliding nuclei, and $s=1,2$ denotes the fragmentation and $\alpha$ a set of intrinsic quantum numbers. Further, $I, l$, and $J$ are the quantum numbers of the total angular momentum, the orbital angular momentum, and the channel spin, respectively. The intrinsic wave function $\Phi_{s \alpha J M}$ describes the collective degrees of freedom and the motion of the extra particle. The transformation of the intrinsic wave function $\Phi_{s \alpha J M}$ to the rotating coordinate system is defined by the relation

$$
\Phi_{s \alpha J M}=\sum_{M^{\prime}} D_{M M^{\prime}}^{J *}\left(\varphi_{s}, \vartheta_{s}, \psi_{s}\right) \tilde{\Phi}_{s \alpha(J) M^{*}} .
$$

The parentheses around the quantum number $J$ of the channel spin indicate that $\tilde{\Phi}$ has a good quantum number $J$ for large separations of the nuclei only. When the nuclei overlap, the extracore nucleon moves in a rotating deformed potential well and, therefore, has no good angular momentum with respect to any center. We use Eq. (24) as the definition of the intrinsic wave function for arbitrary internuclear distances. Inserting Eq. (24) into Eq. (23) we obtain

$$
\begin{aligned}
\psi_{I M}=\sum_{s \alpha l J} & R_{s \alpha l J I}\left(R_{s}\right) i^{l}\left(\frac{2 l+1}{8 \pi^{2}}\right)^{1 / 2} \\
& \times \sum_{M^{\prime}}\left(l 0 J M^{\prime} \mid I M^{\prime}\right) D_{M M^{\prime}}^{I^{*}}\left(\varphi_{s}, \vartheta_{s}, \psi_{s}\right) \tilde{\Phi}_{s \alpha(J) M^{\prime}} .
\end{aligned}
$$

The last equation has been further divided by $1 / \sqrt{2 \pi}$ in order to include the integration over the irrelevant Euler angle $\psi_{s}$ in all matrix elements. Asymptotically $\left(R_{s} \rightarrow \infty\right)$ the radial wave functions approach the usual linear superposition of the Coulomb functions $G_{l}$ and $F_{l}$ multiplied by the $S$ matrix:

$$
\begin{aligned}
& R_{L L_{0} I}\left(R_{s}\right)=J_{L}\left(R_{s}\right) \delta_{L L_{0}}-O_{L}\left(R_{s}\right) S_{L L_{0}}^{I}, \\
& J_{L}, O_{L}=\frac{1}{v_{L}^{1 / 2} R_{s}} \exp \left( \pm i \sigma_{l}\right)\left(G_{l} \mp i F_{l}\right), \\
& L=(s, \alpha, l, J) .
\end{aligned}
$$

In the following, we reorder the wave functions used in Eq. (25) into another set of functions which are more convenient for the rotating coordinate system:

$$
\begin{aligned}
\psi_{I M} & =\sum_{L} R_{L I}\left(R_{s}\right) \varphi_{L I M} \\
& =\sum_{K} R_{K I}\left(R_{S}\right) \psi_{K I M} .
\end{aligned}
$$

Here the orthonormalized channel functions are defined as

$$
\begin{aligned}
\varphi_{L I M}=i^{l}\left(\frac{2 l+1}{8 \pi^{2}}\right)^{1 / 2} \sum_{M^{\prime}} & \left(l 0 J M^{\prime} \mid I M^{\prime}\right) \\
& \times D_{M M^{\prime}}^{I *} \tilde{\Phi}_{s \alpha(J) M^{\prime}} .
\end{aligned}
$$

The index $K$ denotes a new set of quantum numbers, completely defining the wave functions $\psi_{K I M}$ which we will specify in the next sections. The transformation matrix between the two representations is assumed to be independent of the relative coordinate:

$$
A_{L K}^{I}=\left\langle\varphi_{L I M} \mid \psi_{K I M}\right\rangle \text {. }
$$

The $S$ matrix in the $L$ representation, which is needed in the calculation of the cross sections, can be obtained from the relative wave functions $R_{K I}$ in the $K$ representation by two methods:

(a) One defines the asymptotic behavior of 
$R_{K I}$ by that of $R_{L I}$ given in Eq. (26):

$$
R_{K L_{0} I}=\sum_{L}\left(A^{I}\right)_{K \check{L}}^{-1}\left(J_{L} \delta_{L L_{0}}-O_{L} S_{L L_{0}}^{I}\right),
$$

(b) One defines the asymptotic behavior of $R_{K I}$ differently by generalized Coulomb functions and the $S$ matrix, both written in the $K$ representation:

$$
R_{K K_{0} I}=\sum_{K^{\prime}}\left(J_{K K^{\prime}}^{I} \delta_{K^{\prime} K_{0}}-O_{K K^{\prime}}^{I} S_{K^{\prime} K_{0}}^{I}\right),
$$

with

$$
\begin{aligned}
& J_{K K^{\prime}}^{I}=\sum_{L}\left(A^{I}\right)_{K L^{-1}} J_{L} i^{-l} A_{L K^{\prime}}^{I}, \\
& O_{K K^{\prime}}^{I}=\sum_{L}\left(A^{I}\right)_{K L^{-1}} O_{L} i^{l} A_{L K^{\prime}}^{I} .
\end{aligned}
$$

The functions $J_{K K^{\prime}}^{I}$ and $O_{K K^{\prime}}^{I}$ become diagonal in $K$ for $R \rightarrow \infty$, for example, $J_{K K^{\prime}}^{I}(R \rightarrow \infty)=i^{-l} J_{L} \delta_{K K^{\prime}}$. The matrix in the $L$ representation is finally calculated by the transformation

$$
S_{L L^{\prime}}^{I}=\sum_{K K^{\prime}} A_{L K^{\prime}}^{I} S_{K K^{\prime}}^{I}\left(A^{I}\right)_{K^{\prime} L^{\prime}}^{-1} i^{l+l} \cdot
$$

The representation for the wave functions in (27) should be chosen as the set of states classified by $K$ which are selectively excited via the coupling potentials (22). For example, the main coupling potential for the quadrupole vibrations is proportional to $\alpha_{20}^{\prime(i)}$ and, therefore, excites only vibrations with $M=0$ with respect to the intrinsic $z^{\prime}$ axis. Therefore, it is useful to classify the channel wave functions according to the quantum number of $z^{\prime}$ component of angular momentum.

\section{A. Intrinsic wave function for different cores}

In the case of $C_{1} \neq C_{2}$ we do not have to symmetrize the wave function for the exchange of the cores. The antisymmetrization of the extracore nucleon with the cores is currently neglected. The intrinsic wave function $\tilde{\Phi}_{s \alpha(J) M}$ can be expressed as a product of the eigenfunctions of $H_{\text {coll }, i}$ and $h_{\text {ATCSM }}$. These eigenfunctions are defined by the eigenvalue equations:

$$
\begin{aligned}
& H_{\mathrm{col}, i} \psi_{B L M}(i)=E_{\beta L} \psi_{\beta L M}(i), \quad i=1,2 \\
& h_{\mathrm{ATCSM}}\left(\overrightarrow{\mathrm{r}}_{\mathrm{c} . \mathrm{m} .}^{\prime}, s, R_{s}\right) \varphi_{s \lambda(j) m}\left(\overrightarrow{\mathrm{r}}_{\mathrm{c} . \mathrm{m} .}^{\prime}, R_{s}\right) \\
& =\epsilon_{s \lambda(j) ! m !}\left(R_{s}\right) \varphi_{s \lambda(j) m}\left(\overrightarrow{\mathrm{r}}_{\mathrm{c} . \mathrm{m} .}^{\prime}, R_{s}\right) .
\end{aligned}
$$

The wave functions $\psi_{\beta L M}$, classified by the quantum number $\beta$ and angular momentum $L$, describe the collective states of the core nuclei. The matrix elements of the operators $\alpha_{2 \mu}^{\prime(i)}$ between these states can be reduced to the $E 2-$ transition probabilities. ${ }^{13}$ These transition probabilities may be obtained from measurement or directly calculated with realistic nuclear models chosen for $H_{\text {coll }}$ (for collective nuclear models see Refs. 10 and 14).

The wave functions $\varphi_{s \lambda(j) m}$ of the ATCSM depend on the fragmentation $s$ and form an orthonormal set for each value of $s$. The parameters of $h_{\text {ATCSM }}$ have to be so adjusted that the calculated asymptotic single-particle levels agree with the experimental levels of the nucleus with $A_{s}=C_{s}+1$ nucleons. The difference in the mass asymmetries between the two fragmentations is given by

$$
\begin{aligned}
\eta_{1}-\eta_{2} & =\left(C_{1}-C_{2}+1\right) / A-\left(C_{1}-C_{2}-1\right) / A \\
& =2 / A .
\end{aligned}
$$

Since this difference is small, we may insert an averaged mass asymmetry $\bar{\eta}=\left(C_{1}-C_{2}\right) / A$ into the ATCSM. Then the Hamiltonian of the ATCSM and their eigenfunctions and eigenvalues would be independent of the fragmentation $s$. This approximation can be applied in all cases where the asymptotic spectra of the nuclei with $A_{s}=C_{s}+1$ nucleons are sufficiently well reproduced by a single set of parameters of the ATCSM. In the following, however, we formulate the theory without such an approximation.

With the wave functions defined in Eqs. (33) and (34) we construct the following intrinsic wave functions $\tilde{\Phi}$ :

$$
\begin{aligned}
& \tilde{\Phi}_{1 \alpha(J) M}=\left[\left[\psi_{\beta_{1} L_{1}}(1) \otimes \varphi_{1 \lambda(j)}\left(\overrightarrow{\mathrm{r}}_{\mathrm{c} . \mathrm{m} .}^{\prime}, R_{1}\right)\right]^{\left[I_{1}\right]} \otimes \psi_{\beta_{2} L_{2}}(2)\right]_{M}^{[J]}, \\
& 1 \alpha=\left(\beta_{1} L_{1}, \lambda j, I_{1} ; \beta_{2} L_{2}=I_{2}\right), \\
& \tilde{\Phi}_{2 \alpha(J) M}=\left[\psi_{\beta_{1} L_{1}}(1) \otimes\left[\psi_{\beta_{2} L_{2}}(2) \otimes \varphi_{2 \lambda(j)}\left(\overrightarrow{\mathrm{r}}_{\mathrm{c} . \mathrm{m} .}^{\prime}, R_{2}\right)\right]^{\left[I_{2}\right]}\right]_{M}^{[J]}, \\
& 2 \alpha=\left(\beta_{1} L_{1}=I_{1} ; \beta_{2} L_{2}, \lambda j, I_{2}\right) .
\end{aligned}
$$

For large separations the intrinsic wave functions (36) describe the states of the individual nuclei with spins $I_{1}$ and $I_{2}$. The coupled equations are conveniently obtained with the following orthonormalized basis functions:

$$
\begin{aligned}
& \psi_{K I M}=\left(\frac{2 I+1}{8 \pi^{2}}\right)^{1 / 2} D_{M M^{\prime}}^{I^{*}}\left(\varphi_{s}, \vartheta_{s}, \psi_{s}\right) \\
& \times\left[\psi_{\beta_{1} L_{1}}(1) \otimes \psi_{\beta_{2} L_{2}}(2)\right]_{M^{\prime}-m}^{[\Lambda]} \varphi_{s \lambda(j) m}\left(\overrightarrow{\mathrm{r}}_{\mathrm{c} . \mathrm{m} .}^{\prime}, R_{s}\right), \\
& K=\left(s, M^{\prime} ; \beta_{1} L_{1}, \beta_{2} L_{2}, \Lambda ; s \lambda j m\right) .
\end{aligned}
$$

They are eigenfunctions of the Hamiltonian:

$$
\begin{aligned}
& {\left[H_{\mathrm{coll}}+h_{\mathrm{ATCSM}}+\frac{1}{2 \mu_{s} R_{s}{ }^{2}}\left(I^{2}-I_{z}{ }^{2}+J_{\mathrm{coll}}{ }^{2}\right.\right.} \\
& \left.\left.\quad-J_{\mathrm{coll}, \varepsilon^{\prime}}{ }^{2}\right)\right] \psi_{K I M}=E_{K I} \psi_{K I M}, \quad(38 \mathrm{a}) \\
& E_{K I}\left(R_{s}\right)=E_{\beta_{1} L_{1}}+E_{\beta_{2} L_{2}}+\epsilon_{s \lambda(j) \mid m !}\left(R_{s}\right) \\
& +\frac{\hbar^{2}}{2 \mu_{s} R_{s}{ }^{2}}\left(I(I+1)-M^{\prime 2}+\Lambda(\Lambda+1)-\left(M^{\prime}-m\right)^{2}\right) .
\end{aligned}
$$


The transformation needed for the computation of the $S$ matrix and defined in Eq. (28) is given with the wave functions (37) as a sum over Clebsch-Gordan coefficients, which can easily be summed up:

$$
\begin{aligned}
A_{L K}^{I} & =i^{-l}\left(l 0 J M^{\prime} \mid I M^{\prime}\right)\left(\frac{2 l+1}{2 I+1}\right)^{1 / 2}\left\langle\tilde{\Phi}_{s \alpha(J) M^{\prime}} \cdot \mid\left[\psi_{\beta_{1} L_{1}} \otimes \psi_{\beta_{2} L_{2}}\right]_{M^{\prime}{ }_{-m}}^{\left[\Lambda_{j}\right]} \varphi_{s \lambda(j) m}\right\rangle \\
& =i^{-l}(-)^{M^{\prime}}(2 \Lambda+1)^{1 / 2}\left(I-M^{\prime} J M^{\prime} \mid l 0\right)\left(j m \Lambda M^{\prime}-\left.m\right|_{J} J M^{\prime}\right) A_{s}, \\
A_{s=1} & =(-1)^{L_{2}-I_{1}}\left(2 I_{1}+1\right)^{1 / 2}\left\{\begin{array}{ccc}
L_{1} & L_{2} & \Lambda \\
J & j & I_{1}
\end{array}\right\} \\
A_{s=2} & =(-1)^{L_{1}+L_{2}-\Lambda-J}\left(2 I_{2}+1\right)^{1 / 2}\left\{\begin{array}{ccc}
L_{2} & L_{1} & \Lambda \\
J & j & I_{2}
\end{array}\right\}
\end{aligned}
$$

B. Intrinsic wave function for identical cores

An interesting special type of transfer reactions, namely the elastic transfer, occurs for identical cores. Experimental work on elastic transfer reactions has been extensively carried out by von Oertzen et al., ${ }^{5}$ who have analyzed their data in terms of a linear combination of nuclear orbitals (LCNO).

For $C_{1}=C_{2}=C$ we symmetrize the wave function for the exchange of the cores. The total wave function [see Eq. (25)] consists of two parts, the direct and core-exchange term:

$$
\begin{aligned}
\psi_{I M}=\sum_{s \alpha l J M^{\circ}} & i^{l}\left(\frac{2 l+1}{16 \pi^{2}}\right)^{1 / 2}\left(l 0 J M^{\prime} \mid I M^{\prime}\right) \\
& \times\left[R_{s \alpha l J I}\left(R_{s}\right) D_{M M^{\prime}}^{I^{*}}\left(\varphi_{s}, \vartheta_{s}, \psi_{s}\right) \tilde{\Phi}_{s \alpha(J) M^{\circ}}(\text { direct })+R_{s \alpha l J I}\left(\tilde{R}_{s}\right) D_{M M^{\circ}}^{I^{*}}\left(\tilde{\varphi}_{s}, \tilde{\vartheta}_{s}, \tilde{\psi}_{s}\right) \tilde{\Phi}_{s \alpha(J) M^{\circ}} \text { (exchange) }\right) .
\end{aligned}
$$

Here we denote the relative coordinate after core exchange by $\overrightarrow{\tilde{R}}_{s}$. It is given according to Eq. (5) by

$$
\overrightarrow{\tilde{R}}_{1}=-\vec{R}_{2}, \quad \overrightarrow{\tilde{R}}_{2}=-\vec{R}_{1} .
$$

For equal cores the mass asymmetries of the ATCSM have the values $\eta_{1}=-\eta_{2}=1 / A$ 。 As first approximation one may use the symmetric TCSM. Between the wave functions of the ATCSM the following phase relation holds ${ }^{8}$ :

$$
\begin{aligned}
\varphi_{1 \lambda(j) m}\left(x_{\mathrm{c} . \mathrm{m} .}^{\prime},-y_{\mathrm{c} . \mathrm{m} .}^{\prime},\right. & \left.-z_{\mathrm{c} . \mathrm{m} .}^{\prime}, \tilde{R}_{1}\right) \\
= & (-1)^{j-2 m} \varphi_{2 \lambda(j)-m}\left(\overrightarrow{\mathrm{r}}_{\mathrm{c} . \mathrm{m} .}^{\prime}, R_{2}\right) .
\end{aligned}
$$

Using Eqs. (41) and (42) we obtain for the wave function (40)

$$
\begin{gathered}
\psi_{I M}=\sum_{s \alpha l J M^{\prime}} i^{l}\left(\frac{2 l+1}{16 \pi^{2}}\right)^{1 / 2}\left(l 0 J M^{\prime} \mid I M^{\prime}\right) D_{M M^{\prime}}^{I^{*}}\left(\varphi_{s}, \vartheta_{s}, \psi_{s}\right) \\
\quad \times\left[R_{s \alpha l J I}\left(R_{s}\right) \tilde{\Phi}_{s \alpha(J) M^{\prime}}\right. \\
\quad+(-1)^{\left.l-J+I \tilde{s}^{+} L_{s} R_{\tilde{s} \alpha l J I}\left(R_{s}\right) \tilde{\Phi}_{s \tilde{\alpha}(J) M^{\prime}}\right]}
\end{gathered}
$$

with the abbreviations

$$
\begin{aligned}
& \tilde{s}=1 \text { for } s=2 ; \tilde{s}=2 \text { for } s=1 \\
& 1 \alpha=\left(\beta_{1} L_{1}, \lambda j, I_{1} ; \beta_{2} L_{2}\right), \\
& 1 \tilde{\alpha}=\left(\beta_{2} L_{2}, \lambda j, I_{2} ; \beta_{1} L_{1}\right), \\
& 2 \alpha=\left(\beta_{1} L_{1} ; \beta_{2} L_{2}, \lambda j, I_{2}\right), \\
& 2 \tilde{\alpha}=\left(\beta_{2} L_{2} ; \beta_{1} L_{1}, \lambda j, I_{1}\right) .
\end{aligned}
$$

After reordering the summation in Eq. (43) we finally obtain the following expression:

$$
\begin{aligned}
\psi_{I M}=\sum_{s \alpha l J M^{\prime}} & i^{l}\left(\frac{2 l+1}{8 \pi^{2}}\right)^{1 / 2}\left(l 0 J M^{\prime} \mid I M^{\prime}\right) \\
& \times \tilde{R}_{s \alpha l J I}\left(R_{s}\right) D_{M M^{\prime}}^{I^{*}}\left(\varphi_{s}, \vartheta_{s}, \psi_{s}\right) \tilde{\Phi}_{s \alpha(J) M !}
\end{aligned}
$$

with the radial functions

$$
\begin{aligned}
\tilde{R}_{s \alpha l J I}\left(R_{s}\right)= & \frac{1}{\sqrt{2}}\left(R_{s \alpha l J I}\left(R_{s}\right)\right. \\
& \left.+(-1)^{l-J+I s^{+} L_{\tilde{s}}} R_{\tilde{s} \tilde{\alpha} l J I}\left(R_{s}\right)\right) 。
\end{aligned}
$$

For the special case, when both cores are in the ground state $\left(L_{1}=L_{2}=0\right)$, or more generally when $s \alpha=s \tilde{\alpha}$, the radial wave functions for $s=1$ and 2 are related by

$$
\tilde{R}_{1 \alpha l J I}=(-1)^{l-J+I_{1}+L_{2} \tilde{R}_{2 \alpha l J I}} \text { 。 }
$$

Next we symmetrize the wave function in the 
representation defined in Eq. (37) for core exchange. The result is

$$
\psi_{I M}=\sum_{K} \tilde{R}_{K(s) I}\left(R_{s}\right) \psi_{K(s) I M},
$$

where

$$
\begin{aligned}
\tilde{R}_{K(s) I}= & \frac{1}{\sqrt{2}}\left(R_{K(s) I}\left(R_{s}\right)\right. \\
& \left.+(-1)^{I+j+L_{1}+\tilde{L}_{2}-2 M^{\prime}} R_{\tilde{K}(\tilde{s}) I}\left(R_{s}\right)\right), \\
K(s)= & \left(s, M^{\prime} ; \beta_{1} L_{1}, \beta_{2} L_{2}, \Lambda ; s \lambda j m\right), \\
\tilde{K}(\tilde{s})= & \left(\tilde{s},-M^{\prime} ; \beta_{2} L_{2}, \beta_{1} L_{1}, \Lambda ; \tilde{s} \lambda j-m\right) .
\end{aligned}
$$

Similarly to Eq. (47) we find for the case when the cores occupy equal states, $\beta_{1} L_{1}=\beta_{2} L_{2}$ :

$$
\tilde{R}_{K(1) I}=(-1)^{I+j+L 1^{+} L_{2}{ }^{-2 M^{\prime}}} \tilde{R}_{\tilde{K}(2) I} \cdot
$$

Since the difference between the two relative coordinates $\vec{R}_{1}$ and $\vec{R}_{2}$ is small for equal cores, we may approximate the expression (48) by inserting $\vec{R}_{1}=\vec{R}_{2}=\vec{R}$ and using the solutions of the symmetric TCSM which are independent of $s$, namely $\varphi_{s \lambda(j) m} \rightarrow \varphi_{\lambda(j) m}$. The eigenfunctions of the symmetric TCSM have a good parity $\pi= \pm 1$ :

$$
\begin{aligned}
& \psi_{I M}=\left(\frac{2 I+1}{16 \pi^{2}}\right)^{1 / 2} \sum_{K} R_{K I}(R)\left\{D_{M M}^{I *}\left[\psi_{B_{1} L_{1}}(1) \otimes \psi_{B_{2} L_{2}}(2)\right]_{M^{\prime}-m}^{\left[\Lambda^{\prime}\right]} \varphi_{\lambda(j) m}\right. \\
& \left. \pm(-1)^{l}(-1)^{I+j+L_{1^{+}}+L_{2}-2 M^{\prime}} D_{M-M}^{I *} \cdot\left[\psi_{B_{2} L_{2}}(1) \otimes \psi_{\beta_{1} L_{1}}(2)\right]_{-M^{\prime}+m}^{[\Lambda]_{j}} \varphi_{\lambda(j)-m}\right\} .
\end{aligned}
$$

Here $l$ is the orbital angular momentum of the asymptotic STCSM wave functions with respect to their centers. ${ }^{8}$ The structure and symmetry of this wave function are the same as those used in the strong-coupling model (Nilsson model) describing the motion of a nucleon coupled to the rotation and vibrations of a deformed core. ${ }^{10}$

\section{COUPLED CHANNEL EQUATIONS}

In this section we derive coupled channel equations for the radial wave functions. Using the representation defined in Eq. (37) we divide the wave function with respect to the fragmentation:

$$
\begin{aligned}
\psi_{I M}= & \sum_{K(1)} R_{K(1) I}\left(R_{1}\right) \psi_{K(1) I M}\left(\overrightarrow{\mathrm{R}}_{1}, \overrightarrow{\mathrm{r}}_{\mathrm{c} . \mathrm{m} .}^{\prime}, \alpha_{2 \mu}^{\prime(i)}\right) \\
& +\sum_{K(2)} R_{K(2) I}\left(R_{2}\right) \psi_{K(2) I M}\left(\overrightarrow{\mathrm{R}}_{2}, \overrightarrow{\mathrm{r}}_{\mathrm{c} . \mathrm{m} .}^{\prime}, \alpha_{2 \mu}^{\prime(i)}\right) .
\end{aligned}
$$

\section{A. Overlap integrals and shift operators}

Overlap integrals between the two parts of the wave function (52) are, in general, difficult to evaluate, as is known from the exact treatment of recoil effects. The general type of overlap integral is given by

$$
\int \psi_{n}^{*}\left(\overrightarrow{\mathrm{R}}_{1}, \overrightarrow{\mathrm{r}}_{\mathrm{c} . \mathrm{m} .}\right) \psi_{m}\left(\overrightarrow{\mathrm{R}}_{2}, \overrightarrow{\mathrm{r}}_{\mathrm{c} . \mathrm{m} .}\right) d \tau_{1} \text { or } d \tau_{2} \cdot
$$

When the coordinates of integration are chosen as $\overrightarrow{\mathbf{R}}_{s}$ and $\overrightarrow{\mathbf{r}}_{\text {c.m. }}$, the volume element has to be taken as $d \tau_{s}$ [see Eq. (8)]. The operators $S_{1}$ and $S_{2}$, which shift both sets of coordinates, are defined by

$$
\begin{aligned}
& \psi_{m}\left(\overrightarrow{\mathbf{R}}_{2}, \overrightarrow{\mathbf{r}}_{\text {c.m }}\right)=S_{1}\left(\overrightarrow{\mathbf{R}}_{1}, \overrightarrow{\mathbf{r}}_{\text {c.m. }}\right) \psi_{m}\left(\overrightarrow{\mathbf{R}}_{1}, \overrightarrow{\mathbf{r}}_{\text {c.m. }}\right), \\
& \psi_{n}\left(\overrightarrow{\mathbf{R}}_{1}, \overrightarrow{\mathbf{r}}_{\text {c.m. }}\right)=S_{2}\left(\overrightarrow{\mathbf{R}}_{2}, \overrightarrow{\mathbf{r}}_{\text {c.m. }}\right) \psi_{n}\left(\overrightarrow{\mathbf{R}}_{2}, \overrightarrow{\mathbf{r}}_{\text {c.m. }}\right) .
\end{aligned}
$$

These operators fulfill the equation

$$
\begin{aligned}
\int \psi_{n}^{*}\left(\overrightarrow{\mathrm{R}}_{1}, \overrightarrow{\mathbf{r}}_{\mathrm{c} . \mathrm{m} .}\right) S_{1}\left(\overrightarrow{\mathbf{R}}_{1}, \overrightarrow{\mathbf{r}}_{\mathrm{c} . \mathrm{m} .}\right) \psi_{m}\left(\overrightarrow{\mathrm{R}}_{1}, \overrightarrow{\mathbf{r}}_{\mathrm{c} . \mathrm{m} .}\right) d \tau_{1} \\
=\left[\int \psi_{m}^{*}\left(\overrightarrow{\mathrm{R}}_{2}, \overrightarrow{\mathbf{r}}_{\mathrm{c} . \mathrm{m} .}\right) S_{2}\left(\overrightarrow{\mathrm{R}}_{2}, \overrightarrow{\mathbf{r}}_{\mathrm{c} . \mathrm{m} .}\right) \psi_{n}\left(\overrightarrow{\mathrm{R}}_{2}, \overrightarrow{\mathbf{r}}_{\mathrm{c} . \mathrm{m}}\right) d \tau_{2}\right]^{*} .
\end{aligned}
$$

The operators $S_{s}$ can be written in the form of exponential operators, where we have applied Eq. (6):

$$
\begin{aligned}
& S_{1}=\exp \left\{-\frac{1}{\left(C_{2}+1\right) C_{1}}\left[\left(C_{1}-C_{2}\right) \overrightarrow{\mathrm{R}}_{1}+A \overrightarrow{\mathrm{r}}_{\mathrm{c} . \mathrm{m} .}\right] \operatorname{grad}_{1}\right\}, \\
& S_{2}=\exp \left\{\frac{1}{\left(C_{1}+1\right) C_{2}}\left[\left(C_{1}-C_{2}\right) \overrightarrow{\mathrm{R}}_{2}+A \overrightarrow{\mathrm{r}}_{\mathrm{c} . \mathrm{m} .}\right] \operatorname{grad}_{2}\right\} .
\end{aligned}
$$

In principle, the integrals (55) can be calculated numerically. But in the following, we prefer an approximation which takes a Taylor expansion of (56) up to first order in the exponents. If we use the first order terms as they result from the Taylor expansion of (56), Eq. (55) would only be fulfilled approximately to the same order. Since Eq. (55) is important for the proof of the Hermiticity of the coupled equations, we approximate Eq. (56) by the following shift operators which are modified in the constants $\gamma_{1}$ and $\gamma_{2}$ so that they satisfy Eq. (55) exactly:

$$
S_{s}\left(\overrightarrow{\mathrm{R}}_{s}, \overrightarrow{\mathrm{r}}_{\mathrm{c} . \mathrm{m} .}\right)=1+\gamma_{s}\left[\left(C_{1}-C_{2}\right) \overrightarrow{\mathrm{R}}_{s}+A \overrightarrow{\mathrm{r}}_{\mathrm{c} . \mathrm{m}} .\right] \operatorname{grad}_{s},
$$

where

$$
\begin{aligned}
& \gamma_{1}=-\left(\alpha^{2}+\alpha \beta+\beta^{2}\right) / 3 \alpha^{3}, \\
& \gamma_{2}=\left(\alpha^{2}+\alpha \beta+\beta^{2}\right) / 3 \beta^{3}, \\
& \alpha=\left(C_{1}+1\right) C_{2} ; \beta=C_{1}\left(C_{2}+1\right) .
\end{aligned}
$$


The gradients operate on the relative coordinates $\vec{R}_{1}$ and $\vec{R}_{2}$. After the transformation to the rotating coordinate system we get the following equation:

$$
\left[\left(C_{1}-C_{2}\right) \overrightarrow{\mathrm{R}}_{s}+A \overrightarrow{\mathrm{r}}_{\mathrm{c} . \mathrm{m} .}\right] \operatorname{grad}_{s}=\left[\left(C_{1}-C_{2}\right) R_{s}+A z_{\mathrm{c} . \mathrm{m} .}^{\prime}\right] \frac{\partial}{\partial R_{s}}+\frac{i}{\hbar} \frac{A}{R_{s}}\left[x_{\mathrm{c} . \mathrm{m} .}^{\prime}\left(I_{s}-J-J_{\mathrm{coll}}\right)_{y^{\circ}}-y_{\mathrm{c} . \mathrm{m} . .}^{\prime}\left(I_{s}-J-J_{\text {coll }}\right)_{x^{\prime}}\right],
$$

where

$$
\overrightarrow{\mathrm{J}}=\overrightarrow{\mathrm{r}}_{\mathrm{c} . \mathrm{m} .}^{\prime} \times \overrightarrow{\mathrm{p}}_{\mathrm{c} . \mathrm{m} .}^{\prime}+\overrightarrow{\mathrm{s}}^{\prime} .
$$

In the following we use the approximations (57) instead of the exact expressions (56).

\section{B. Coupled equations}

The Hamiltonian $H$, as given in Eq. (18), depends on the fragmentation $s$. If $H$ were the exact microscopic Hamiltonian, both representations would fulfill the identity relation with the shift operator (56):

$$
\begin{aligned}
S_{1}\left(\overrightarrow{\mathrm{R}}_{1}, \overrightarrow{\mathrm{r}}_{\mathrm{c} . \mathrm{m} .}\right) H\left(2, \overrightarrow{\mathrm{R}}_{2}=\overrightarrow{\mathrm{R}}_{1}, \overrightarrow{\mathrm{r}}_{\mathrm{c} . \mathrm{m} .}, \alpha_{2 \mu}\right) \\
=H\left(1, \overrightarrow{\mathrm{R}}_{1}, \overrightarrow{\mathrm{r}}_{\mathrm{c} . \mathrm{m} .}, \alpha_{2 \mu}\right) S_{1}\left(\overrightarrow{\mathrm{R}}_{1}, \overrightarrow{\mathrm{r}}_{\mathrm{c} . \mathrm{m} .}\right) .
\end{aligned}
$$

For the realistic Hamiltonians $(s=1,2)$, defined in Eq. (18), Eq. (60) is satisfied for the kinetic energy exactly. However, the potential energies fulfill Eq. (60) only approximately, since they are represented by models in the
Hamiltonian (18). Also, when we insert the approximative expressions (57) for the shift operators in Eq. (60), this equation cannot be exactly satisfied. Because Eq. (60) is important for the proof of the Hermiticity of the coupled equations, we replace these operators by their average:

$$
\left.\begin{array}{l}
S_{s}\left(\overrightarrow{\mathrm{R}}_{s}, \overrightarrow{\mathrm{r}}_{\mathrm{c} . \mathrm{m} .}\right) H\left(\tilde{s}, \overrightarrow{\mathrm{R}}_{\tilde{s}}=\overrightarrow{\mathrm{R}}_{s}, \ldots\right) \\
H\left(s, \overrightarrow{\mathrm{R}}_{s}, \ldots\right) S_{s}\left(\overrightarrow{\mathrm{R}}_{s}, \overrightarrow{\mathrm{r}}_{c . \mathrm{m} .}\right)
\end{array}\right\} \rightarrow H S\left(s, \overrightarrow{\mathrm{R}}_{s}, \ldots\right),
$$

where $H S$ is defined by $(s=1,2)$

$$
\begin{aligned}
H S\left(s, \overrightarrow{\mathrm{R}}_{s}, \ldots\right)=\frac{1}{2}\left[S_{s}\left(\overrightarrow{\mathrm{R}}_{s}, \overrightarrow{\mathrm{r}}_{\mathrm{c} . \mathrm{m} .}\right) H\left(\tilde{s}, \overrightarrow{\mathrm{R}}_{\tilde{s}}=\overrightarrow{\mathrm{R}}_{s}, \ldots\right)\right. \\
\left.+H\left(s, \overrightarrow{\mathrm{R}}_{s}, \ldots\right) S_{s}\left(\overrightarrow{\mathrm{R}}_{s}, \overrightarrow{\mathrm{r}}_{\mathrm{c} . \mathrm{m} .}\right)\right]
\end{aligned}
$$

The averaged operators $H S(s)$ have the same Hermitian property such as the shift operators in Eq. (55):

$$
\int \psi_{n}^{*}\left(\overrightarrow{\mathrm{R}}_{1}, \ldots\right) H S\left(1, \overrightarrow{\mathrm{R}}_{1}, \ldots\right) \psi_{m}\left(\overrightarrow{\mathrm{R}}_{2}=\overrightarrow{\mathrm{R}}_{1}, \ldots\right) d \tau_{1}=\left(\int \psi_{m}^{*}\left(\overrightarrow{\mathrm{R}}_{2}, \ldots\right) H S *\left(2, \overrightarrow{\mathrm{R}}_{2}, \ldots\right) \psi_{n}\left(\overrightarrow{\mathrm{R}}_{1}=\overrightarrow{\mathrm{R}}_{2}, \ldots\right) d \tau_{2}\right)^{*},
$$

where $\psi_{n}$ and $\psi_{m}$ are wave functions describing different fragmentations. We note that the Hamiltonians are real except the imaginary potentials which have been introduced in Eq: (12). With the approximation (62) we obtain the following system of coupled channel equations for the radial functions:

$$
\begin{aligned}
& \sum_{K^{\prime}(1)} D_{K(1), K^{\prime}(1)}\left(R_{1}\right) R_{K^{\prime}(1) I}\left(R_{1}\right)+\sum_{K^{\prime}(2)} D_{K(1), K^{\prime}(2)}\left(R_{1}\right) R_{K^{\prime}(2) I}\left(R_{1}\right)=0, \\
& \sum_{K^{\prime}(2)} D_{K(2), K^{\prime}(2)}\left(R_{2}\right) R_{K^{\prime}(2) I}\left(R_{2}\right)+\sum_{K^{\prime}(1)} D_{K(2), K^{\prime}(1)}\left(R_{2}\right) R_{K^{\prime}(1) I}\left(R_{2}\right)=0 .
\end{aligned}
$$

The first sum in these equations describes elastic scattering and inelastic excitation, and the second sum describes the transfer reactions. The differential operators are calculated by integrating over the Euler angles, the single-particle and collective coordinates, but not over the radial coordinate $R_{s}$ :

$$
\begin{aligned}
& D_{K(s), K^{\prime}(s)}\left(R_{s}\right)= \int \psi_{K}^{*}(s) I M \\
& D_{K(s), K^{\prime}(\tilde{s})}\left(\overrightarrow{\mathrm{R}}_{s}, \overrightarrow{\mathrm{r}}_{\mathrm{c} . \mathrm{m} .}^{\prime}, \alpha_{2 \mu}^{\prime(i)}\right)\left(H\left(s, \overrightarrow{\mathrm{R}}_{s}, \ldots\right)-E\right) \psi_{K^{\prime}(s) I M}\left(\overrightarrow{\mathrm{R}}_{s}, \overrightarrow{\mathrm{r}}_{\mathrm{c} . \mathrm{m} .}^{\prime}, \alpha_{2 \mu}^{\prime(i)}\right) d \Omega_{s} d^{3} \gamma_{\mathrm{c} . \mathrm{m} .}^{\prime} d \tau_{\alpha_{2 \mu}^{\prime}(s) I M}\left(\overrightarrow{\mathrm{R}}_{s}, \overrightarrow{\mathrm{r}}_{\mathrm{c} . \mathrm{m} .}^{\prime}, \alpha_{2 \mu}^{\prime(i)}\right)\left(H S\left(s, \overrightarrow{\mathrm{R}}_{s}, \ldots\right)-E S_{s}\left(\overrightarrow{\mathrm{R}}_{s}, \overrightarrow{\mathrm{r}}_{\mathrm{c} . \mathrm{m}}^{\prime}\right) \psi_{K^{\prime}(\tilde{s}) I M}\left(\overrightarrow{\mathrm{R}}_{\tilde{s}}=\overrightarrow{\mathrm{R}}_{s}, \overrightarrow{\mathrm{r}}_{\mathrm{c} . \mathrm{m} .}^{\prime}, \alpha_{2 \mu}^{\prime(i)}\right)\right. \\
& \times d \Omega_{s} d^{3} r_{\mathrm{c} \mathrm{m} \cdot}^{\prime} d \tau_{\alpha_{2 \mu}^{\prime}},
\end{aligned}
$$

where $H(s)$ is defined in Eq. (18), $H S(s)$ in Eq. (62), and $S_{s}$ in Eq. (57). The same equations result for the symmetrized wave functions (48) in the case of identical cores.

\section{Evaluation of the differential operators}

The differential operators, defined in Eq. (65), are most easily obtained if we are reminded that the wave functions $\psi_{K I M}$ are eigensolutions of the Hamiltonian $H_{0}(s)$ [see Eq. (38)]: 


$$
H_{0}(s)=H_{\text {coll }}+h_{\text {ATCSM }}(s)+\frac{1}{2 \mu_{s} R_{s}{ }^{2}}\left(I^{2}-I_{z}{ }^{2}+J_{\text {coll }}{ }^{2}-J_{\text {coll }, z^{\prime}}{ }^{2}\right) .
$$

Therefore, we divide the total Hamiltonian (18) into the following parts:

$$
H(s)=-\frac{\hbar^{2}}{2 \mu_{s} R_{s}}\left(\frac{\partial}{\partial R_{s}}+D_{s}\right)^{2} R_{s}+U_{0}\left(R_{s}\right)+i W\left(R_{s}, \overrightarrow{\mathrm{I}}^{2}, E\right)+H_{0}(s)+W_{\text {rot }}(s)+W_{\text {coup }}(s),
$$

where $W_{\text {rot }}$ is defined by

$$
\begin{gathered}
W_{\text {rot }}(s)=\frac{1}{2 \mu_{s} R_{s}^{2}}\left[\frac{1}{2}\left(J_{s}^{+} J_{s}^{-}+J_{s}^{-} J_{s}^{+}\right)-I_{s}^{+}\left(J_{s}^{-}+J_{\text {coll }}^{-}\right)-I_{s}^{-}\left(J_{s}^{+}+J_{\text {coll }}^{+}\right)+J_{s}^{+} J_{\text {coll }}^{-}+J_{s}^{-} J_{\text {coll }}^{+}\right] . \\
\text {1. Matrix elements } D_{K / s), K^{\prime}(s)}
\end{gathered}
$$

These matrix elements are given by, according to Eqs. (65a) and (67),

$$
\begin{aligned}
D_{K(s), K^{\prime}(s)=} & \delta_{K(s), K(s)}\left[-\frac{\hbar^{2}}{2 \mu_{s} R_{s}} \frac{d^{2}}{d R_{s}^{2}} R_{s}+U_{0}\left(R_{s}\right)+i W\left(R_{s}, I(I+1) \hbar^{2}, E\right)+E_{K I}-E\right] \\
& -\frac{\hbar^{2}}{2 \mu_{s}}\left[\left\langle\psi_{K(s) I M}\left|\left(\frac{\partial}{\partial R_{s}}+D_{s}\right)^{2}\right| \psi_{K^{\prime}(s) I M}\right\rangle+\frac{2}{R_{s}}\left\langle\psi_{K(s) I M}\left|\left(\frac{\partial}{\partial R_{s}}+D_{s}\right)\right| \psi_{K^{\prime}(s) I M}\right\rangle \frac{d}{d R_{s}} R_{s}\right] \\
& +\left\langle\psi_{K(s) I M}\left|W_{\text {rot }}+W_{\text {coup }}\right| \psi_{K^{\prime}(s) I M}\right\rangle .
\end{aligned}
$$

The matrix elements with the operator $\partial / \partial R_{s}+D_{s}$ are typical for molecular wave functions and cause the radial coupling. They can be reduced to matrix elements between the ATCSM wave functions and vanish for $R_{s} \rightarrow \infty$ :

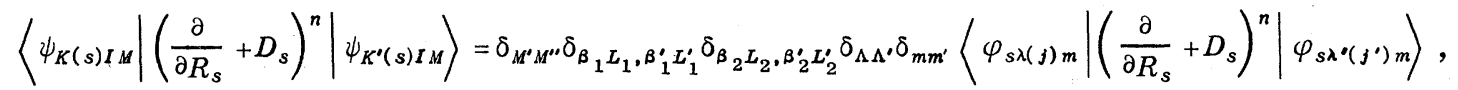

$n=1,2$.

The matrix elements of $W_{\text {rot }}$ are caused by the rotation of the intrinsic coordinate system. They are straightforwardly reduced to the following form:

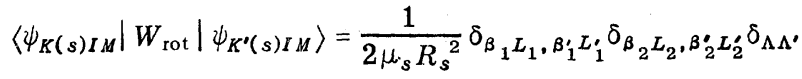

$$
\begin{aligned}
& \times\left(\delta_{M^{\prime} M^{\prime \prime}} \delta_{m m^{\prime}}\left\langle\varphi_{s \lambda(j) m}\left|\frac{1}{2}\left(J_{s}^{+} J_{s}^{-}+J_{s}^{-} J_{s}^{+}\right)\right| \varphi_{s \lambda^{\prime}\left(j^{\prime}\right) m}\right\rangle\right. \\
& -\delta_{\lambda(j), \lambda^{\prime}\left(j^{\prime}\right)} \delta_{m m^{\prime}} \hbar^{2}\left\{\delta_{M^{\prime \prime}, M^{\prime}+1}\left[\left(I+M^{\prime}+1\right)\left(I-M^{\prime}\right)\left(\Lambda+M^{\prime}-m+1\right)\left(\Lambda-M^{\prime}+m\right)\right]^{1 / 2}\right. \\
& \left.+\delta_{M^{\prime \prime}, M^{\prime}-1}\left[\left(I-M^{\prime}+1\right)\left(I+M^{\prime}\right)\left(\Lambda-M^{\prime}+m+1\right)\left(\Lambda+M^{\prime}-m\right)\right]^{1 / 2}\right\} \\
& +\delta_{m^{\prime}, m-1} \hbar\left\{\delta_{M^{\prime \prime} M^{\prime}}\left[\left(\Lambda+M^{\prime}-m+1\right)\left(\Lambda-M^{\prime}+m\right)\right]^{1 / 2}-\delta_{M^{\prime \prime}, M^{\prime}-1}\left[\left(I-M^{\prime}+1\right)\left(I+M^{\prime}\right)\right]^{1 / 2}\right\} \\
& \times\left\langle\varphi_{s \lambda(j) m}\left|J_{s}^{+}\right| \varphi_{s \lambda^{\prime}\left(j^{\circ}\right) m-1}\right\rangle \\
& +\delta_{m^{\prime}, m+1} \hbar\left\{\delta_{M^{\prime \prime} M^{\prime}}\left[\left(\Lambda-M^{\prime}+m+1\right)\left(\Lambda+M^{\prime}-m\right)\right]^{1 / 2}-\delta_{M^{\prime \prime}, M^{\prime}+1}\left[\left(I+M^{\prime}+1\right)\left(I-M^{\prime}\right)\right]^{1 / 2}\right\} \\
& \left.\times\left\langle\varphi_{s \lambda(j) m}\left|J_{s}^{-}\right| \varphi_{s \lambda^{\prime}\left(j^{\prime}\right) m+1}\right\rangle\right) \text { 。 }
\end{aligned}
$$

Only asymptotically $\left(R_{s} \rightarrow \infty\right)$, when $j$ becomes a good quantum number, the matrix elements of the operators $J_{s}$ reduce to the usual simple expressions

$$
\begin{aligned}
& \left\langle\varphi_{s \lambda(j) m}\left|\frac{1}{2}\left(J_{s}^{+} J_{s}^{-}+J_{s}^{-} J_{s}^{+}\right)\right| \varphi_{s \lambda^{\prime}\left(j^{\prime}\right) m}\right\rangle \underset{R_{s} \rightarrow \infty}{=} \delta_{\lambda_{j}, \lambda^{\prime} j^{\prime}}\left(j(j+1)-m^{2}\right) \hbar^{2}, \\
& \left\langle\varphi_{s \lambda(j) m}\left|J_{s}^{ \pm}\right| \varphi_{s \lambda^{\prime}\left(j^{\prime}\right) m \mp 1}\right\rangle \underset{R_{s} \rightarrow \infty}{=} \delta_{\lambda j, \lambda^{\prime} j^{\prime}}[(j \mp m+1)(j \pm m)]^{1 / 2} \hbar .
\end{aligned}
$$

$$
\text { 2. Matrix elements } D_{K(s), K^{\prime}(\widetilde{s})}
$$

These matrix elements, defined in Eq. (65b), contain the recoil effects in the transfer reactions. For convenience, we separate the recoil contributions out of the transfer matrices: 


$$
\begin{aligned}
D_{K(s), K^{\prime}(\tilde{s})}= & D_{K(s), K^{\prime}(\tilde{s})}^{T}+D_{K(s), K^{\prime}(\tilde{s})}^{R} \\
= & \left\langle\psi_{K(s) I M}\left|\frac{1}{2}\left(H\left(1, \overrightarrow{\mathrm{R}}_{s}, \ldots\right)+H\left(2, \overrightarrow{\mathrm{R}}_{s}, \ldots\right)\right)-E\right| \psi_{K^{\prime}(\tilde{s}) I M}\right\rangle \\
& +\left\langle\psi_{K(s) I M}\left|\frac{1}{2}\left\{\left(S_{s}-1\right) H\left(\tilde{s}, \overrightarrow{\mathrm{R}}_{s}, \ldots\right)+H\left(s, \overrightarrow{\mathrm{R}}_{s}, \ldots\right)\left(S_{s}-1\right)\right\}-\left(S_{s}-1\right) E\right| \psi_{K^{\prime}(\tilde{s}) I M}\right\rangle .
\end{aligned}
$$

If all recoil effects are neglected, the second term in Eq. (73) has to be disregarded. For the first term we find the expression

$$
\begin{aligned}
D_{K(s), K^{\prime}(\tilde{s})=}^{T}= & \delta_{M^{\prime} M^{\prime \prime}} \delta_{m m^{\prime}} \delta_{\beta_{1} L_{1}, \beta_{1}^{\prime} L_{1}^{\prime} \delta_{\beta_{2}} L_{2}, \beta_{2}^{\prime} L_{2}^{\prime} \delta_{\Lambda \Lambda^{\prime}}} \\
\times & \left(\langle \varphi _ { s \lambda ( j ) m } | \varphi _ { \tilde { s } \lambda ^ { \prime } ( j ^ { \prime } ) m } \rangle \left\{-\frac{\hbar^{2}}{4}\left(\frac{1}{\mu_{1}}+\frac{1}{\mu_{2}}\right) \frac{1}{R_{s}} \frac{d^{2}}{d R_{s}{ }^{2}} R_{s}+U_{0}\left(R_{s}\right)\right.\right. \\
& \left.+i W\left[R_{s}, I(I+1) \hbar^{2}, E\right]+\frac{1}{2}\left(E_{K(s) I}+E_{K^{\prime}(\tilde{s}) I}\right)-E\right\} \\
& -\frac{\hbar^{2}}{4}\left[\left\langle\varphi_{s \lambda(j) m}\left|\frac{1}{\mu_{1}}\left(\frac{\partial}{\partial R_{s}}+D_{1}\right)^{2}+\frac{1}{\mu_{2}}\left(\frac{\partial}{\partial R_{s}}+D_{2}\right)^{2}\right| \varphi_{\tilde{s} \lambda^{\prime}\left(j^{\prime}\right) m}\right\rangle\right. \\
& \left.\left.+\frac{2}{R_{s}}\left\langle\varphi_{s \lambda(j) m}\left|\frac{1}{\mu_{1}}\left(\frac{\partial}{\partial R_{s}}+D_{1}\right)+\frac{1}{\mu_{2}}\left(\frac{\partial}{\partial R_{s}}+D_{2}\right)\right| \varphi_{\tilde{s} \lambda^{\prime}\left(j^{\prime}\right) m}\right\rangle \frac{d}{d R_{s}} R_{s}\right]\right) \\
& +\frac{1}{2}\left\langle\psi_{K(s) I M}\left|\sum_{s=1}^{2}\left[W_{\text {rot }}(s)+W_{\text {coup }}(s)\right]\right| \psi_{K^{\prime}(\tilde{s}) I M}\right\rangle
\end{aligned}
$$

The matrix elements of $W_{\text {rot }}$ can be calculated in complete analogy to Eq. (71). The overlap matrix elements $\left\langle\varphi_{s} \mid \varphi_{\tilde{s}}\right\rangle$ are different from zero in the reaction zone where we use different ATCSM for the two fragmentations. It would be a realistic approximation to use the same ATCSM for both fragmentations with an average mass asymmetry $\bar{\eta}=\left(C_{1}-C_{2}\right) / A$ [see Eq. (35)], independent of $s$. In that case, the overlap matrix elements $\left\langle\varphi_{s} \mid \varphi_{\tilde{s}}\right\rangle$ are identically zero since the solutions of the same ATCSM are orthonormal. This approximation needs a redefinition of the operators $D_{s}$ and $J_{s}$ in order to ensure that special matrix elements, such as Eq. (70), vanish asymptotically. A forthcoming paper will discuss this approach in detail. ${ }^{15}$

For the second term in (73) we first consider the matrix elements of the shift operator (57) which we express in spherical tensor operators:

$$
\begin{aligned}
S_{s}\left(\overrightarrow{\mathrm{R}}_{s}, \overrightarrow{\mathrm{r}}_{\mathrm{c} . \mathrm{m} .}\right)=1+\gamma_{s}\left\{\left[\left(C_{1}-C_{2}\right) R_{s}+A z_{\mathrm{c} . \mathrm{m} .}^{\prime}\right] \frac{\partial}{\partial R_{s}}+\frac{A}{\hbar}\left(\frac{2 \pi}{3}\right)^{1 / 2} \frac{\gamma_{\mathrm{co} \mathrm{m}_{e}}^{\prime}}{R_{s}}\left[Y_{1-1}\left(\vartheta_{\mathrm{c}, \mathrm{m} .}^{\prime}, \varphi_{\mathrm{c} . \mathrm{m} .}^{\prime}\right)\left(I_{s}^{+}-J^{+}-J_{\mathrm{coll}}^{+}\right)\right.\right. \\
\left.\left.+Y_{11}\left(\vartheta_{\mathrm{c} . \mathrm{m} .}^{\prime}, \varphi_{\mathrm{c} . \mathrm{m} .}^{\prime}\right)\left(I_{s}^{-}-J^{-}-J_{\mathrm{coll}}^{-}\right)\right]\right\} .
\end{aligned}
$$

With this expression we obtain

$$
\begin{aligned}
& \left(S_{s}-1\right)_{K(s), K^{\prime}(\tilde{s})}=\left\langle\psi_{K(s) I M}\left|S_{s}\left(\overrightarrow{\mathrm{R}}_{s}, \overrightarrow{\mathrm{r}}_{\mathrm{c} . \mathrm{m} .}\right)-1\right| \psi_{K^{\prime}(\tilde{s}) I M}\right\rangle
\end{aligned}
$$

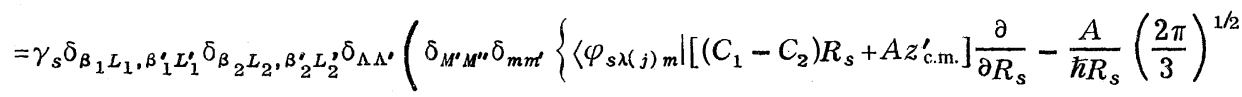

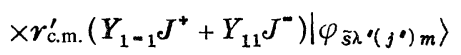

$$
\begin{aligned}
& \left.+\left\langle\varphi_{s \lambda(j) m}\left|\left(C_{1}-C_{2}\right) R_{s}+A z_{\mathrm{c} . \mathrm{m} \cdot}^{\prime}\right| \varphi_{\tilde{s} \lambda^{\prime}(j) m}\right\rangle \frac{d}{d R_{s}}\right\} \\
& +\frac{A}{R_{s}}\left(\frac{2 \pi}{3}\right)^{1 / 2}\left(\delta_{m t^{\prime}, m+1}\left\langle\varphi_{s \lambda(j) m}\left|\gamma_{\mathrm{c} . \mathrm{m} .}^{\prime} Y_{1-1}\right| \varphi_{\tilde{s} \lambda^{\circ}\left(j^{\prime}\right) m+1}\right\rangle\right.
\end{aligned}
$$

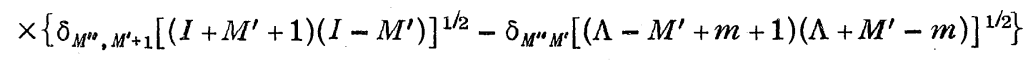

$$
\begin{aligned}
& +\delta_{m^{\prime}, m-1}\left\langle\varphi_{s \lambda(j) m}\left|\gamma_{\mathrm{c} . \mathrm{m} .}^{\prime} Y_{11}\right| \varphi_{\tilde{s} \lambda^{\prime}\left(j^{\prime}\right) m-1}\right\rangle \\
& \left.\times\left\{\delta_{M^{\prime \prime}, M^{\prime}-1}\left[\left(I-M^{\gamma}+1\right)\left(I+M^{\prime}\right)\right]^{1 / 2}-\delta_{M^{\prime \prime} M^{\prime}}\left[\left(\Lambda+M^{\prime}-m+1\right)\left(\Lambda-M^{\prime}+m\right)\right]^{1 / 2}\right\}\right) .
\end{aligned}
$$

The operator $\left(S_{s}-1\right)_{K K^{\prime}}$ vanishes asymptotically $\left(R_{s} \rightarrow \infty\right)$. If $j$ were a good quantum number, the matrix 
elements would have had the selection rule $j^{\prime}=j, j \pm 1$. With the result (76) we may write the matrix elements of the second part in Eq. (73):

$$
\begin{aligned}
& D_{K(s), K^{\prime}(\tilde{s})=}^{R}\left(S_{s}-1\right)_{K K^{\prime}}\left(-\frac{\hbar^{2}}{2 \mu_{\tilde{s}}} \frac{1}{R_{s}} \frac{d^{2}}{d R_{s}{ }^{2}} R_{s}+U_{0}\left(R_{s}\right)+i W\left(R_{s}, \ldots\right)+E_{K^{\prime}(\xi) I}-E\right) \\
&+\frac{1}{2}\left(-\frac{\hbar^{2}}{2 \mu_{s}} \frac{1}{R_{s}} \frac{d^{2}}{d R_{s}{ }^{2}} R_{s}+U_{0}\left(R_{s}\right)+i W\left(R_{s}, \ldots\right)+E_{K(s) I}-E\right)\left(S_{s}-1\right)_{K K^{\prime}}+\text { rest. }
\end{aligned}
$$

Here the rest term contains the operators $W_{\text {rot }}$ and $W_{\text {coup }}$ and the remainder of the radial kinetic energy. In order to obtain cross sections for elastic, inelastic, and transfer processes, computational work to solve the coupled equations (64) is required. As a first step we have further simplified the equations (64) using the same ATCSM for both fragmentations. This approach will be published in Ref。 15 .

\section{CONCLUDING REMARKS}

In this paper we have developed a practicable method to use molecular single-particle wave functions also for transfer reactions. The singleparticle states depend on the fragmentations which are different in the direct and transfer channels. For first numerical calculations it would be sufficient to apply the same ATCSM in all fragmentations. This approximation has the unique advantage that all single-particle states become orthogonal independently of the fragmentation. Thereby, the transfer operators are greatly simplified。

There exist mainly two possibilities in choosing the intrinsic basis functions. In Refs。 4 and 8 we have taken $M$-weighted sums over the ATCSM states as basis states for the coupled equations so that these equations are asymptotically decoupled. This basis has the disadvantage that the states are no longer eigenstates of the ATCSM. In this paper we have used the individual eigenfunctions of the ATCSM as basis states. However, the resulting equations are still coupled asymptotically by the Coriolis potential. Both choices of bases are completely equivalent as long as the same sets of ATCSM states are taken into account. The method of this paper has the advantage that the channels for excitation and transfer can be classified by the individual levels of the ATCSM. Therefore, it is most transparent how to select out the ATCSM levels for the coupled channel equations. The disadvantage of this basis lies in its asymptotic coupling and, as discussed, can be easily overcome by a simple matrix multiplication in the calculation of the $S$ matrix.

The present theory can be straightforwardly extended to the case of more than one extracore nucleon. As an example for application we mention the ${ }^{13} \mathrm{C}-{ }^{13} \mathrm{C}$ scattering. Terlecki et al. ${ }^{8}$ have calculated the elastic scattering and inelastic excitation of the neutrons within the framework of the molecular theory. The next steps which need to be carried out are the calculation of the neutron transfer cross sections ${ }^{13} \mathrm{C}+{ }^{13} \mathrm{C} \rightarrow{ }^{12} \mathrm{C}+{ }^{14} \mathrm{C}$, and the inelastic excitation of the ${ }^{12} \mathrm{C}$ cores. The formalism of the antisymmetrization of the extracore neutrons leads to additional relative coordinates for each partition of the extracore nucleons. Since the antisymmetrization represents an exchange of nucleons, it generates similar effects in the coupled channel equations as the physical transfer processes.

It is worthwhile to develop a theory of particlehole excitations in the molecular formalism (see also Ref. 5). In such a theory the inelastic scattering of even-even nuclei can be described without violating the Pauli principle in contrast to the particle-core model where the antisymmetrization between the core and extracore nucleons is completely neglected. The residual nucleon-nucleon interaction could be partly taken into account by using Hartree-Fock solutions ${ }^{16}$ for the single-particle states instead of the ATCSM states.

The cross sections for the excitation and transfer of nucleons in reactions, such as ${ }^{13} \mathrm{C}+{ }^{16} \mathrm{O}$ $\rightarrow{ }^{12} \mathrm{C}+{ }^{17} \mathrm{O}$, should exhibit certain enhancements as function of the heavy ion energies which arise due to avoided level crossings in the two-center level diagrams. ${ }^{17}$ Similar effects have been observed in atomic physics and are known as promotion processes according to Fano and Lichten. ${ }^{18}$ It would be a unique signature for the formation of nuclear molecular orbits if the promotion process for nucleons could be detected in the transfer and excitation cross sections ${ }^{19}$ Up until now, no experimental investigations in that direction have been known to us, which can give an answer to the important problem of the existence of molecular orbits in nuclear heavy ion collisions.

This work was supported by the Deutsche Forschungsgemeinschaft, the Bundesministerium für Forschung und Technologie and the Gesellschaft für Schwerionenforschung (GSI)。 
${ }^{1}$ Proceedings of the International Conference on Resonances in Heavy-Ion Reactions, Hvar, Yugoslavia 1977, edited by N. Cindro (North-Holland, Amsterdam, 1978).

${ }^{2}$ P. Holzer, U. Mosel, and W. Greiner, Nucl. Phys. A138, 241 (1969), D. Scharnweber, U. Mosel, and W. Greiner, ibid. A164, 257 (1971); J. Maruhn and W. Greiner, Z. Phys. 251, 431 (1972); M. G. Mustafa, U. Mosel, and H. W. Schmitt, Phys. Rev. Lett. 28, 1536 (1972); Phys. Rev. C 7,1519 (1973).

${ }^{3}$ K. Pruess and W. Greiner, Phys. Lett. 33B, 197 (1970); U. Mosel, T. D. Thomas, and P. Riesenfeldt, Phys. Lett. 33B, 565 (1970).

${ }^{4}$ J. Y. Park, W. Scheid, and W. Greiner, Phys. Rev. C $\underline{6}$, 1565 (1972).

${ }^{5}$ W. von Oertzen and W. Nörenberg, Nucl. Phys. A207, 113 (1973); W. von Oertzen and H. F. Bohlen, Phys. Rep. 19C, 1 (1975).

${ }^{6}$ F. Becker, S. Joffily, C. Beccaria, and G. Baron, Nucl. Phys. A221, 475 (1974).

${ }^{7}$ A. V. Matveenko and I. Lovas, Nucl. Phys. A299, 333 (1978).

${ }^{8} \mathrm{G}$. Terlecki and W. Scheid, in Proceedings of the Informal Workshop on Heavy Ion Scattering, Strasbourg, 1.976 , edited by F. Becker, (Universite Louis Pasteur,
Strasbourg, 1976), p. VIII-1; G. Terlecki, W. Scheid, H. F. Fink, and W. Greiner, Phys. Rev. C 18, 265 (1978).

${ }^{9}$ K. Pruess, Nucl. Phys. A278, 124 (1977); K. Pruess and P. Lichtner, ibid. A291, 475 (1977).

${ }^{10}$ See M. Eisenberg and $\mathrm{W}$. Greiner, Nuclear Theory, Vol.1, Nuclear Models (North-Holland, Amsterdam, 1970).

${ }^{11} \mathrm{~J}$. Maruhn and W. Greiner, Z. Phys. 251, 431 (1972).

${ }^{12}$ H. J Fink, W. Scheid, and W. Greiner, J. Phys. G 1 , 685 (1975).

${ }^{13}$ H. J. Fink, W. Scheid, and W. Greiner, Nucl. Phys. A188, 259 (1972).

${ }^{14}$ G. Gneuss and W. Greiner, Nucl. Phys. A171, 449 (1971).

${ }^{15}$ J. Y. Park et al. (unpublished).

${ }^{16}$ H. Flocard, Phys. Lett. $\underline{49 B}, 129$ (1974); P. G. Zint and U. Mosel, Phys. Rev. C 14, 1488 (1976); H. Chandra and U. Mosel, Nucl. Phys. A298, 151 (1978).

${ }^{17} \mathrm{~J} . \mathrm{Y}$. Park et al. (unpublished).

${ }^{18}$ For a review of the promotion process see M. Barat and W. Lichten, Phys. Rev. A $\underline{6}, 211$ (1972).

${ }^{19} \mathrm{C}$. Toepffer, private communication. 